\title{
Consulta de ENFERMAGEM EM SAÚde MENTAL NA ATENÇÃO PRIMÁRIA EM SAÚDE
}

\author{
Eduarda Berckenbrock Bolsoni ${ }^{1}$ \\ Isabella Paola Meirstelin Heusy ${ }^{1}$ \\ Zenandia Feliciano da Silva ${ }^{I}$ \\ Maria Itayra Padilha ${ }^{2}$ \\ Jeferson Rodrigues ${ }^{3}$
}

\begin{abstract}
O objetivo, nesta pesquisa, foi compreender a importância da consulta de enfermagem em saúde mental na Atenção Primária à Saúde. Utilizou-se abordagem qualitativa do tipo descritiva, cujos participantes foram sete enfermeiros que compõem a Equipe de Estratégia de Saúde da Família, vinculados a um Centro de Saúde. Os dados foram obtidos por entrevista e grupo de discussão. Todos os enfermeiros consideraram a consulta de enfermagem em saúde mental importante por ser uma estratégia de acompanhamento, pelo fortalecimento do vínculo com o paciente na longitudinalidade do cuidado, por ampliar a participação do enfermeiro perante as discussões de caso com a equipe multiprofisssional e contribuir para a resolutividade dos casos de saúde mental.
\end{abstract}

Descritores: Enfermagem; Atenção Primária à Saúde; Saúde Mental.

\footnotetext{
${ }^{1}$ Alunos de graduação em enfermagem, Universidade Federal de Santa Catarina (UFSC), Florianópolis, SC, Brasil.

2 PhD, Professor Titular, Departamento de Enfermagem, Universidade Federal de Santa Catarina (UFSC), Florianópolis, SC, Brasil.

${ }^{3}$ PhD, Professor Adjunto, Departamento de Enfermagem, Universidade Federal de Santa Catarina (UFSC), Florianópolis, SC, Brasil.
}

\section{Correspondência}

Jeferson Rodrigues

Servidão Cristiano Wanderley Faria, no 60, apto 703. Bairro Trindade.

CEP: 88.040-405, Florianópolis, SC, Brasil

Email: jeferson.rodrigues@ufsc.br 


\section{MENTAL HEALTH NURSING CONSULTATIONS IN PRIMARY HEALTH CARE}

The aim of this research was to understand the importance of mental health nursing consultation in Primary Health Care. A qualitative, descriptive approach was used with seven participants; the nurses who made up the Family Health Care Strategy Team linked to a Health Care Center. Data were obtained from interviews and from a discussion group. All of the nurses considered mental health nursing consultation important as a monitoring strategy, for strengthening the link with the patient throughout the care, for extending nursing participation in case discussions with the multi-professional health team and because it contributes to resolving mental health cases.

Descriptors: Nursing; Primary Health Care; Mental Health.

\section{CONSUlTA DE ENFERMERÍA EN SALUd MENTAL EN LA ATENCIÓN PRIMARIA DE SALUd}

La finalidad en esta investigación fue comprender la importancia de la consulta de enfermería en salud mental en la Atención Primaria de Salud. Fue utilizada aproximación cualitativa del tipo descriptiva, cuyos participantes fueron siete enfermeros miembros del Equipo de Estrategia de Salud de la Familia, vinculados a un Centro de Salud. Los datos fueron recolectados por entrevista y grupo de discusión. Todos los enfermeros consideraron la consulta de enfermería en salud mental importante por ser una estrategia de acompañamiento, debido al fortalecimiento del vínculo con el paciente en la longitudinalidad del cuidado, por ampliar la participación del enfermero ante las discusiones de caso con el equipo multiprofesional y contribuir a la resolutividad de los casos de salud mental.

Descriptores: Enfermería; Atención Primaria de Salud; Salud Mental.

\section{Introdução}

No Brasil, a Reforma Psiquiátrica se fortaleceu a partir da década de 1990, com a inclusão de ações e serviços de saúde mental nas políticas públicas de saúde. Com a aprovação da Lei $n^{\circ} 10.216$, houve a criação do Centro de Atenção Psicossocial e Serviços Residenciais Terapêuticos, significando uma relação de alta complexidade para média complexidade ${ }^{(1)}$. A Atenção Primária em Saúde (APS), de acordo com o momento histórico, político e social da saúde do Brasil, não foi diretamente investida em ações de saúde mental, quanto a outros níveis de complexidade. Sua evolução passou por três momentos significativos. O primeiro momento, entre 1980 e 2000, destaca algumas experiências de inserções de equipes mínimas de saúde mental nas Unidades Básicas de Saúde que incluíam psicólogos, psiquiatras e assistentes sociais. No segundo momento, de 2001 a 2008, foram criadas as diretrizes nacionais para saúde mental na Atenção Básica. A partir de 2001, ocorreram oficinas de consensos técnicos entre Coordenação Nacional de Saúde Mental, Departamento de Atenção Básica e Seminário Internacional sobre Saúde Mental. O terceiro momento aconteceu a partir de 2008 até os dias atuais, quando ocorreu a institucionalização das ações de saúde mental na Atenção Primária, com implantação nos Núcleos de Apoio à Saúde da Família. O matriciamento 
se torna um dispositivo fundamental para a ampliação do acesso e acompanhamento nesse nível de atenção ${ }^{(2)}$.

A APS é porta de entrada aos usuários para o acesso aos serviços de saúde do sistema público. Desse modo, a APS deve estar fundamentada nos princípios do Sistema Único de Saúde (SUS) como universalidade, acessibilidade, vínculo, longitudinalidade do cuidado, integralidade, responsabilização, humanização, equidade e participação social. A gestão e a prática profissional guiada por esses princípios proporcionam atendimento à saúde com qualidade à população ${ }^{(3)}$. A Estratégia de Saúde da Família (ESF), que atua na Atenção Primária à Saúde, tem investido na promoção da saúde e prevenção da doença, e avançado na melhoria da qualidade de vida, incluindo as pessoas com transtornos mentais ${ }^{(4)}$.

Destarte, uma das formas do avanço no cuidado, com vistas aos princípios do SUS pelo enfermeiro na Atenção Primária, é a consulta de enfermagem em saúde mental, que traz autonomia, reconhecimento profissional e oportunidade de interação com o indivíduo em sofrimento e a sua família. Sendo a consulta de enfermagem considerada uma das ações que melhor representa o profissional da categoria, permite que a assistência ocorra de maneira sistemática e que as necessidades básicas afetadas possam ser identificadas. Pode ser realizada sob forma de problemas de enfermagem, de forma individual, sendo possível planejar, executar e avaliar a assistência prestada a cada pessoa ${ }^{(5)}$. Nesse contexto, o estudo teve como questão de pesquisa: qual a importância da consulta de enfermagem em saúde mental na Atenção Primária à Saúde? O objetivo geral do estudo foi compreender a importância da consulta de enfermagem em saúde mental na Atenção Primária à Saúde.

\section{Método}

Optou-se pela abordagem de pesquisa qualitativa do tipo descritiva. Para obtenção dos dados, foram utilizados dois tipos de técnicas de pesquisa, a entrevista semiestruturada e grupos de discussão. $\mathrm{O}$ estudo foi realizado no município de Florianópolis, Santa Catarina, no qual os participantes do estudo foram sete enfermeiros que compõem a Equipe de Estratégia de Saúde da Família, lotados em um Centro de Saúde (CS). Esse número contemplou a totalidade dessa categoria profissional no CS estudado. Os enfermeiros participantes da pesquisa possuíam idade entre 26 e 43 anos, a maioria do sexo feminino, com tempo de graduação de 3 a 8 anos, e que trabalhavam no mesmo Centro de Saúde, com variação de 2 meses a 3 anos. Em relação à formação pós-graduada um não a possuía, cinco enfermeiros eram especialistas em saúde da família e um mestre em saúde pública.

As entrevistas foram realizadas mediante agendamento com os profissionais e no local de trabalho desses. Foram realizados dois grupos de discussão com os mesmos enfermeiros entrevistados, de modo a possibilitar uma visão coletiva do objeto estudado. Essa técnica possibilita compreender o objeto de estudo por meio do diálogo entre as pessoas do agrupamento, escolhidas intencionalmente pelo pesquisador ${ }^{(6)}$.

Os critérios de inclusão dos participantes do estudo foram: estarem vinculados ao serviço e presentes durante o tempo da pesquisa em campo. As entrevistas e os grupos de discussão foram realizados no $\mathrm{CS}$, no período de abril a maio de 2014, buscando-se sigilo e ambientes reservados para que as informações ficassem restritas às pesquisadoras. A análise de conteúdo foi utilizada para o tratamento dos dados ${ }^{(7)}$. Após as entrevistas no campo, essas foram transcritas. Os dados foram organizados e, após a realização da leitura, os dados foram interpretados e decodificados, buscando-se os códigos em comum para elaboração da categorização. A investigação seguiu o rigor ético da pesquisa com seres humanos e foi submetida e aprovada pelo Comitê de Ética em Pesquisa da Universidade Federal de Santa Catarina (UFSC), sob $\mathrm{n}^{\circ} 538.836$.

\section{Resultados e Discussões}

Realização da consulta de enfermagem em saúde mental na atenção primária à saúde

A realização da consulta como atividade indispensável de enfermagem, para promover a 
saúde, está diretamente ligada à oferta de cuidado na atenção primária. Em saúde mental, essa contribui como estratégia na longitudinalidade do cuidado na APS e amplia o conjunto de atividades na elaboração do Projeto Terapêutico Singular (PTS).

DeacordocomaPortaria/SS/GAB/n²6/2014, da Prefeitura Municipal de Florianópolis, Art. 21, todos os profissionais, incluindo o enfermeiro, durante o horário de trabalho, devem realizar atendimentos de demanda espontânea e de demanda programada/cuidado continuado ${ }^{(8)}$. Dentre as atribuições do enfermeiro previstas na Carteira de Serviços da Atenção Primária de Florianópolis, além do atendimento da demanda espontânea e programada, o profissional enfermeiro deve realizar a classificação de risco/vulnerabilidade, prestando atendimentos clínicos/cirúrgicos de urgência/emergência à pessoa em sofrimento psíquico agudo/grave. $\mathrm{Na}$ atenção domiciliar, deve prestar atendimento aos usuários com transtorno mental com dificuldade de socialização/isolamento e/ou má adesão ao tratamento $^{(8)}$.

A demanda programada é a consulta de enfermagem, específica do processo de trabalho do enfermeiro que contribui para uma atuação mais eficaz. Dessa forma, a assistência de forma sistemática possibilita o conhecimento daquele que recebe o cuidado, obtendo subsídios para auxiliar a autonomia do usuário, de forma que as necessidades básicas possam ser identificadas dentro da singularidade da pessoa, de maneira individualizada $^{(9)}$. Diante dos relatos abaixo, pode-se perceber que a consulta não é realizada em dias específicos para cada especificidade, como saúde da mulher, saúde da criança, saúde do idoso, entre outros, mas de acordo com a demanda, como é mostrado a seguir.

A gente não tem horário voltado para nenhum tipo de consulta, a gente não tem reserva de horário para nada, a gente deixa espontânea a marcação... Então nunca aconteceu assim de ter uma consulta só para sofrimento psíquico, só para saúde mental, sabe? [...] pacientes que chegam na demanda espontânea que eu identifico que é de saúde mental, a gente normalmente marca retorno para próxima semana comigo... (E3).

Programada em saúde mental acaba não fazendo muito, é mais demanda espontânea, procura quando está em alguma crise ou para uma situação que necessita fazer acompanhamento, émais com psicólogo, psiquiatra mesmo [...] Seria mais consultas para renovação de receita, essas coisas, não para acompanhamento em saúde mental, é bem pouca a demanda para enfermeiro [...] (E1).

Era um baixo risco a gente medicou e só pediu para ela voltar dali 5 dias... para uma nova consulta e ai ela voltou comigo de novo, a programada, ela já estava melhor, mas ela precisou ser medicada e aí a gente encaminhou para uma psicóloga junto, então foi só desta vez, e depois a gente ficou com o matriciamento da psicóloga... (E6).

Os enfermeiros descrevem que não realizam a consulta de enfermagem de forma programada em saúde mental por não haver marcação de consulta com esse tipo de demanda. Ainda referem que observam, em outros tipos de atendimentos, a saúde mental do usuário, e se sentem preparados para realizar a consulta. O usuário é orientado para retornar à consulta programada e, se houver necessidade, matriciamento ou encaminhamento para os profissionais do Núcleo de Apoio à Saúde da Família (NASF).

A elaboração do planejamento da consulta de enfermagem em saúde mental, junto à equipe de apoio, compõe a identificação da necessidade de cuidados de enfermagem da pessoa ou da família, comunidade, propondo ações estratégicas próprias que auxiliam na promoção da saúde ${ }^{(10)}$.

Importância da consulta de enfermagem em saúde mental na atenção primária à saúde

$\mathrm{Na}$ atenção primária, a consulta de enfermagem em saúde mental é uma ação privativa do enfermeiro que prioriza a pessoa expressar suas dificuldades, identificá-las e buscar a resolutividade em conjunto com o profissional. Além disso, o enfermeiro é o suporte para a pessoa buscar sua recuperação. A enfermagem atua na saúde mental das pessoas, visando o equilíbrio das emoções, adaptações e níveis de estresse $^{(9)}$. A função do enfermeiro é de agente terapêutico que tem, como base da consulta, o relacionamento terapêutico estabelecido com a pessoa. A proposta do modelo psicossocial de atenção passa da centralidade do atendimento médico para as necessidades do sujeito, requerendo um tipo de ação profissional que supere os domínios profissionais. O enfermeiro é desafiado a disponibilizar seu saber técnico e uma habilidade extra com a imprevisibilidade e a diversidade do cotidiano ${ }^{(9)}$.

A consulta de enfermagem é uma ferramenta 
importante para o atendimento em saúde mental, pois auxilia o enfermeiroa entendere compreender o indivíduo na sua face individual e também de forma coletiva e familiar, ajudando, assim, o desenvolvimento de ações que repercutirão sobre o usuário, família e comunidade ao qual está inserido ${ }^{(11)}$. Na consulta, os enfermeiros promovem, restauram, mantêm e reabilitam a saúde mental, tanto dos indivíduos quanto das famílias e comunidades. As suas habilidades são baseadas na atenção psicossocial, teorias de personalidade e comportamento humano ${ }^{(9)}$.

Eu acho importante até para acompanhar a evolução do paciente... se a gente atende um paciente o primeiro dia hoje, e estabelece um plano de ações para ele a gente precisa fazer consultas, tá vendo se aquilo que planejamos para ele se tá dando certo, se ele tá seguindo, e se a gente pode futuramente remanejar ações... (E4).

Ela estava chorando um monte, a gente acolheu. Vou agendar uma consulta contigo para tu retornar, tu aceitas? Daí ela assim: então tá, semana que vem eu venho na consulta [...] Então tem muito paciente que vincula contigo, quer ir sempre contigo, e é tu que tem que atender, tu não vai ficar falando vai lá com a psicóloga, vai lá... Então tu tem que saber atender (E3).

Eu acho assim, é uma equipe, a enfermagem faz parte. Então é de grande importância ela estar participando desse processo. A consulta de enfermagem é importante para estabelecer o vínculo e não ficar o vínculo só com o médico, mas ficar com a equipe... (E5).

A consulta de enfermagem é uma ferramenta privativa do enfermeiro que serve para o acompanhamento de grupos de atenção, como gestante, criança e idoso. No entanto, a consulta de enfermagem em saúde mental, como forma de acompanhamento, ainda é incipiente. A importância da consulta de enfermagem em saúde mental se destaca mediante a oportunidade do cuidado singular à pessoa em sofrimento, da autonomia conquistada pela categoria profissional e da atuação e resolutividade do enfermeiro como integrante da equipe multiprofissional.

A assistência em saúde mental consiste na relação de troca entre o generalista e o especialista, esse acaba sendo um desafio atual na relação ensino/serviço. O especialista deve estar disponível para desenvolver a troca de conhecimento com o generalista, essa troca de saberes, realizada entre os profissionais, é necessária para que haja a supervisão e o crescimento mútuo da equipe, proporcionando cuidado humanizado e eficaz.
Etapas da consulta de enfermagem em saúde mental na atenção primária à saúde

Para que ocorra a sistematização da consulta de enfermagem na APS, é necessário que etapas sejam seguidas para arealização da consulta, como modo de organização do processo de trabalho e sistematização de cuidado integral. As etapas para realização da consulta de enfermagem em saúde mental são: anamnese ou histórico do indivíduo, exame psíquico, exame físico (diferencial da consulta comumente proposta), comentários/ observações do enfermeiro, diagnósticos de enfermagem, proposta terapêutica ou prescrição de enfermagem. A anamnese, ou histórico, do indivíduo é composta pelos itens: identificação, queixa principal, história da doença atual, história patológica pregressa, história social, história familiar, necessidade de cuidado e nível de autonomia ${ }^{(12-13)}$.

A comunicação terapêutica é essencial na anamnese, pois proporciona abertura entre profissional e usuário, para que o segundo se sinta confortável para expressar suas queixas, sentimentos e emoções. Esse é um momento que o profissional tem a oportunidade de conhecer, compreender e saber aspectos da história da pessoa que interferem no seu modo de viver e agir. $\mathrm{O}$ exame psíquico abrange dados de avaliação das funções psíquicas e nele o enfermeiro deve avaliar: apresentação geral, linguagem e pensamento, sensopercepção, afetividade e humor, atenção e concentração, memória, orientação e consciência ${ }^{(14) .}$

O exame físico é considerado uma importante ferramenta, pois a pessoa em sofrimento psíquico apresenta patologias físicas com maior frequência que o restante da população. Esse exame pode ser uma forma de aproximação do usuário e profissional, quando realizado de maneira respeitosa. A sequência deve ser seguida de forma cefalocaudal, as etapas são inspeção, palpação, percussão e ausculta ${ }^{(12)}$.

Depois de realizadas as etapas acima, surgem os comentários e observações do enfermeiro, momento em que são colocadas as situações percebidas pelo profissional como, por exemplo, relacionamento familiar, comportamentos do indivíduo, aparência, emoções, sentimentos, 
impressões que o enfermeiro teve ao avaliar esse sujeito $^{(12)}$. É a oportunidade de o enfermeiro se colocar de acordo com seu conhecimento e ideias, podendo fazer alguns questionamentos, demonstrar para a pessoa o seu envolvimento, empatia e se colocar à disposição para ajudá-lo a compreender suas aflições.

O diagnóstico de enfermagem é obtido por meio da análise dos dados coletados. Alguns enfermeiros preferem usar formulário para consulta com diagnósticos preestabelecidos, outros preferem espaços que listam os problemas levantados e para cada um deles é planejada uma ou mais intervenções. A proposta terapêutica ou prescrição de enfermagem é o conjunto de intervenções ou ações a serem desenvolvidas pelo próprio enfermeiro, a equipe de enfermagem e a equipe multiprofissional ${ }^{(12)}$.

As intervenções definidas podem ser tanto individuais quanto grupais, para o indivíduo e até mesmo sua família. É necessário, para cada intervenção proposta, objetivos, metas e prazos a serem cumpridos. A avaliação desse processo é contínua e, de acordo com o resultado, devem ser buscadas novas estratégias, novos encaminhamentos ou manter a conduta empregada $^{(12)}$. Um dos entrevistados desenvolveu como estratégia para a consulta a anamnese, segue o relato.

A primeira coisa é a identificação das pessoas, paciente e profissional, a coleta de dados, o histórico, ouvir o paciente e saber todas as demandas dele... o exame fisico, análise, a interconsulta e a gente vai estar fechando ou fazendo encaminhamentos [...]. Eu faço um roteiro já com demanda de saúde mental. Eu deixo ele falando e vou anotando os pontos principais, todos são pontos importantes e vou trabalhando junto com ele e a gente vai planejando o cuidado dele... (E5).

Percebe-se, nessa fala, que o entrevistado faz uma sistematização da consulta de enfermagem. Tal procedimento é importante para a organização do serviço e cada profissional deve buscar capacitação para ter segurança do atendimento prestado, buscando, junto à pessoa com sofrimento psíquico, a maneira adequada de corresponsabilidade para a adesão ao cuidado.

A pessoa fala da queixa dela, direcionando com algumas perguntas e vou fazendo o histórico, depois, se necessário, faço exame físico e converso com o profissional necessário, se for necessário encaminhar para psicóloga, ou com o médico para fazer encaminhamento para o CAPS [...]. Eu fico mais no S, no SOAP, no O é mais queixa de saúde mental, não faço todo o exame físico. A questão é análise, $P[\ldots]$. Fico mais no organizar os pontos que me listou, no $S[\ldots]$ (E3).

As etapas da consulta de enfermagem em saúde mental são as mesmas realizadas nas consultas de enfermagem em geral, percebese ênfase maior na escuta para identificar o sofrimento psíquico e o exame psíquico, com o propósito de elaborar um plano de cuidados que contemple as necessidades e o interesse da pessoa. Como processo de trabalho, a consulta de enfermagem independente da sua área de atuação (saúde mental, ginecologia, obstetrícia, gerontologia, entre outros), observa a mesma estrutura e pode ser usada na prática em diversos momentos ${ }^{(14)}$. Considera-se que, para a consulta de enfermagem em saúde mental, é necessário o estabelecimento de ambiente adequado onde haja privacidade e não ocorram interrupções, pois, dessa forma, a pessoa se sente à vontade para expor suas queixas. No exame físico, é preciso estar atento se os sinais e sintomas estão relacionados às condições fisiológicas ou psíquicas da pessoa. Outro diferencial relatado é a escuta e a relação terapêutica onde não se pode estar sempre no lugar daquele que fala ou escuta, é preciso trocar de lugar. Escutar o outro significa ser capaz de deixar sua emoção encontrar sua expressividade. $\mathrm{O}$ enfermeiro deve se mostrar ao outro como um ser consciente e sensível ${ }^{(15-16)}$.

\section{Limites pessoais para realização da consulta de enfermagem em saúde mental}

Para que a consulta de enfermagem em saúde mental seja realizada, é necessário que os limites pessoais dos profissionais sejam reconhecidos, estabelecendo metas para que esses limites sejam, também, superados. O enfermeiro escuta histórias de vida parecidas com as suas, ou de alguém de sua família, e, dessa forma, acaba observandose no outro e criando um misto de emoções entre o profissional e o pessoal. Os sentimentos e as impressões dos enfermeiros podem ser produto de muitos fatores, como a experiência clínica profissional, aspectos transferenciais e contratransferenciais, preconceitos, valores pessoais que são carregados de maneira inevitável, no entanto, muitas vezes são produtos 
de intuições que, moldadas pelo estudo ou pelo amadurecimento da prática clínica, podem levar a ações terapêuticas ${ }^{(12)}$.

No processo de comunicação e relacionamento interpessoal, os indivíduos compartilham experiências, ideias e sentimentos, com subjetividade de ambos. Quando o enfermeiro consegue, de modo consciente, reconhecer suas emoções durante esse relacionamento, sendo elas consideradas positivas ou não, pode-se controlar, ao contrário da repressão, e utilizá-las de modo intencional e positivo nas interações. Quando se dá o passo de envolvimento emocional, certamente os relacionamentos se tornam mais autênticos e verdadeiros, sendo o que se deseja no cuidado no atual contexto ${ }^{(17)}$. A subjetividade permeia a interação que é fruto da prática profissional, da qual emergem fatores que vão além do exame físico, porque diz respeito às características do sujeito e suas particularidades. Quando o sujeito causa inquietação no profissional de saúde, além de sua mente consciente, mobiliza emoções, sendo despertados saberes, gestos e emoções com capacidade para esclarecimentos e potencial terapêutico $^{(18-19)}$.

Eu tenho dificuldade de atender casos de saúde mental geralmente quando me mobiliza. Isso é uma coisa para estudar um pouco. Porque se a gente não está resolvida com o mesmo problema, de repente você vai confundir, será que você vai precisar... Fica muito no senso comum e não na parte cientifica profissional da saúde mental (E6).

A gente já discutiu algumas vezes que é a tal da contrarreferência eu acho. Tem um nome de quando tu bota no outro tu lembra de alguém, tu lembra da tua vó, tu lembra do teu pai [...] (E3).

Quando tu não te sente bem pra atender o paciente o melhor que tu tem a fazer é delegar para outra pessoa. Tu tem que ser o porto seguro dessa pessoa, tem que passar uma segurança pra ela, tem que ser o profissional, tens que se vestir (E5).

O envolvimento emocional, relatado pelos profissionais, é importante diante do relacionamento terapêutico, apesar de ser descrito como dificuldade para o atendimento em saúde mental. O fato de se relacionar com o outro, ouvi-lo com atenção, pode despertar sentimentos e emoções no profissional que não precisam ser reprimidos, mas controlados e expressados pelo mesmo, de modo que não guarde tais sentimentos ou se sinta prejudicado, para que ocorra um cuidado científico e humanizado ao sujeito.
Conquanto, é necessário que o profissional procure alternativas para expor seus anseios, buscar autoconhecimento, já que também esses são dotados de sentimentos, inseguranças e preocupações, comuns aos profissionais da área da saúde. Esse sentimento do profissional de humanidade pelo outro, inserindo-se nas experiências de sofrimento mental relatados pela pessoa, favorece a capacidade de escutar com empatia, olhar o indivíduo como semelhante e, despido de julgamentos, ter sua própria compreensão como pessoa e profissional ${ }^{(14)}$. Mesmo considerando as emoções e sentimentos inerentes aos profissionais como comum à nossa existência, há relatos em que não foi possível lidar com o caso da outra pessoa, não estando seguro para ser resolutivo. Diante dos limites pessoais, é essencial que o enfermeiro compreenda se possui condições para realizar o atendimento a pessoa, refletindo seus próprios limites, capacidades e motivações, reconhecendo e aceitando quando não apresenta condições psicológicas para atuar em determinadas situações ${ }^{(14)}$.

\section{Considerações Finais}

A complexidade que envolve o contexto estudado da consulta de enfermagem está na relação entre dois campos de práticas e gestão do cuidado: a atenção primária e a saúde mental. A intersecção entre eles é fortalecida quando a identificação da demanda em saúde mental não seja percebida como uma fragmentação de um cuidado integral para um cuidado especialista. A relação entre a atenção primária e a saúde mental propõe que o cuidado seja relacional e colaborativo, pois são dois campos de conhecimento que estão em relação, onde um não se sobrepõe ao outro.

O estudo alcançou o objetivo de compreender a importância da consulta de enfermagem em saúde mental na Atenção Primária à Saúde. Os relatos dos participantes do estudo consideraram a consulta de enfermagem em saúde mental como importante instrumento para o estabelecimento e fortalecimento de vínculo com a pessoa em sofrimento psíquico e para o acompanhamento longitudinal do cuidado à pessoa com transtorno mental. 
O contexto que envolve a consulta de enfermagem é permeado pela organização do trabalho em saúde da unidade que inclui atendimentoà demanda espontânea e programada. No atendimento do enfermeiro, na modalidade demanda espontânea, cuja necessidade envolve saúde mental, a pessoa é acolhida por meio da escuta qualificada, empatia e respeito. Sendo assim, caso o enfermeiro identifique que há necessidade de acompanhamento, é agendada a consulta de enfermagem voltada à saúde mental como estratégia de retorno. Um dos limites observados na pesquisa foi a fragilidade dos enfermeiros em apontarem saberes que possam ampliar o conhecimento do campo da saúde mental, nas consultas de enfermagem com demandas específicas.

\section{Referências}

1. Brasil. Lei $n^{\circ} 10.216$, de 6 de abril de 2001 . Dispõe sobre a proteção e os direitos das pessoas portadoras de transtornos mentais e redireciona o modelo assistencial em saúde mental. [acesso em 2013 nov 22]. Disponível em: http://www. planalto.gov.br/ccivil_03/leis/leis_2001/I10216. htm.

2. Pôrto KF. Elementos para uma política de avaliação das ações de saúde mental na atenção primária: contribuições de uma pesquisa qualitativa avaliativa [dissertação]. Campinas (SP): Universidade Estadual de Campinas, Departamento de Saúde Coletiva; 2012.

3. Ministério da Saúde (Org.). Cadernos de Atenção Básica: Acolhimento à demanda espontânea: Queixas mais comuns na Atenção Básica. v. 2. $1^{\mathrm{a}}$ ed. Brasília, 2013. [Acesso em: 06 jun. 2014]. Disponível em: http://bvsms. saude.gov.br/bvs/publicacoes/acolhimento_ demanda_espontanea_queixas_comuns.pdf.

4. Ministério da Saúde (BR). Reforma Psiquiátrica e política de saúde mental no Brasil. Conferência Regional de Reforma dos Serviços de Saúde Mental: 15 anos depois de Caracas. Brasília (DF): OPAS; 2005. Disponível em: http://bvsms.saude.gov.br/bvs/publicacoes/ Relatorio15_anos_Caracas.pdf.

5. Mendes MH, Freitas VA, Gomes ET. Consulta de enfermagem: uma prática necessária aos indivíduos com transtornos mentais. Rev.
Enfermagem Integrada 2009 Jul [acesso 2014 jun 12];2(1):225-37. Disponível em: http:// www.unilestemg.br/enfermagemintegrada/ artigo/v2/Mariana_mendes_Valeria_freitas_e_ Everton_gomes.pdf.

6. Callejo J. El grupo de discussión: introdución a una práctica de investigación. Barcelona: Editorial Ariel; 2001.

7. Minayo MCS. O desafio do conhecimento. $11^{\mathrm{a}}$ ed. São Paulo (SP); Hucitec; 2008.

8. Prefeitura Municipal de Florianópolis. Secretária Municipal da Saúde. Portaria da Secretaria Municipal de Saúde $\mathrm{n}^{\circ} 26$ de 23 de março de 2014 [acesso 2014 jun 11]. Disponível em: https://docs.google.com/file/ d/OBxvFvjfrlbywLXZfemh4TzFxT00/edit.

9. Tavares CMM. Atuação do enfermeiro de saúde mental no modo de atenção psicossocial. In: Santos I, David HMSL, Silva D, Tavares CMM. Enfermagem e campos de prática em saúde coletiva - realidade, questões e soluções. Rio de Janeiro: Atheneu; 2008. p. 220-39.

10. Gaspa, EMM. Consulta de enfermagem de saúde mental e psiquiátrica em contexto domiciliário [dissertação]. Sintra (PT): Universidade Católica Portuguesa, Departamento de Instituto de Ciência da Saúde; 2011.

11. Canabrava DS, Vilela JC, Brusamarelo T, Roehrs H, Maftum MA. Consulta de enfermagem em saúde mental sustentada na teoria das relações interpessoais: relato de experiência. Ciência, Cuidado e Saúde 2011;10(1):150-6. Disponível em: http://periodicos.uem.br/ojs/index.php/ CiencCuidSaude/article/view/8044/pdf.

12. Bressan VR. Consulta de Enfermagem: sistematização voltada ao projeto terapêutico. In: Marcolan JF, Castro RCBR. Enfermagem em saúde mental e psiquiátrica: Desafios e possibilidades do novo contexto do cuidar. Rio de Janeiro: Elsevier; 2013. p. 281-298.

13. Fornazier ML, Siqueira MM. Consulta de enfermagem a pacientes alcoolistas em um programa de assistência ao alcoolismo. J. Bras. Psiquiatr. 2006;55(4):280-7. Disponível em: http://www.scielo.br/scielo.php?pid=S0047$20852006000400004 \& \mathrm{script}=\mathrm{sci}$. abstract\&tlng=pt.

14. Gonçalves JRL, Ferreira LA, Ferreira PCS. As interfaces das linhas de cuidados e a saúde mental. In: Santos AS, Cubas MR. 
Saúde Coletiva: linhas de cuidado e consulta de enfermagem. Rio de Janeiro: Elsevier; 2012. p. 235-260.

15. Bondan RMM. Consulta de enfermagem em saúde mental: sob a perspectiva da Teorista Hildegard Peplau [dissertação]. Rio Grande: Universidade Federal do Rio Grande; 2006.

16. Fernandes MA, Sousa LEN, Sousa AR, Evangelista MF. Cuidados de enfermagem ao portador de transtorno afetivo bipolar: relato de experiência. Rev. Enfer. UFPI 2012;1(2):135-8.

17. Ghiorzi AR. O quotidiano dos trabalhadores em saúde. Texto Contexto-Enferm-2003, outdez, 12(4): 551-8.

18. Castro RCBR. Comunicação e relacionamento terapêuticos nos encontros do cuidado em saúde mental. In: Marcolan JF, Castro RCBR. Enfermagem em saúde mental e psiquiátrica: Desafios e possibilidades do novo contexto do cuidar. Rio de Janeiro: Elsevier; 2013. p.19-34.

19. Amarante AL, Lepre AS, Gomes JLD, Pereira AV, Dutra VFD. As estratégias dos enfermeiros parao cuidadoemsaúdemental no programa saúde da família. Texto Contexto-Enferm. 2011 Mar; 20(1): 85-93. Disponível em: http://www.scielo. br/scielo.php?script $=$ sci_arttext\&pid $=$ S010407072011000100010\&lng=pt. http://dx.doi. org/10.1590/S0104-07072011000100010 